\title{
Polymeric Surface Alteration via Scanning Probe Microscopy
}

\author{
Jolanta A. Watson *, Christopher L. Brown ${ }^{\dagger}$, Sverre Myhra ${ }^{*}$ and Gregory S. Watson ${ }^{\ddagger}$ \\ * Nanoscale Science and Technology Centre \\ Griffith University, Kessels Road, Nathan QLD 4111, Australia \\ Email: jolanta.watson@griffith.edu.au \\ Telephone: (617) 3735-7531, Fax: (617) 3735-7656 \\ ${ }^{\dagger}$ School of Science, Nanoscale Science and Technology Centre \\ Griffith University, Kessels Road, Nathan QLD 4111, Australia \\ Email: c.l.brown@griffith.edu.au \\ \# Department of materials, Oxford University, Begbroke Park, UK \\ Email: Sverre.Myhra@materials.ox.edu.au \\ ‡ School of Science, Nanoscale Science and Technology Centre \\ Griffith University, Kessels Road, Nathan QLD 4111, Australia \\ Email: g.watson@griffith.edu.au
}

\begin{abstract}
Polymeric materials which are used in the manufacture of biosensors must satisfy requirements for performance, specificity and biocompatibility, particularly with in vivo medical applications. This study focuses on the physical alteration/manipulation of three polymer surfaces (Polyimide, PDMS and P('BuMA)), via the Scanning Probe Microscope (SPM). A clean incompressible silicon surface was used for calibration purposes. Manipulation (particularly on the softer polymer surfaces, PDMS and P('BuMA)) was achieved using stiff levers (i.e., $>4 \mathrm{nNnm}^{-1}$ ). The resultant alteration was then analyzed using a soft lever $\left(<<1 \mathrm{nNnm}^{-1}\right)$ to avoid further manipulation and alteration. The polymeric materials were selected because of their varying degree of 'stiffness' (i.e., Young's modulus), commercial availability, potential application as low cost biosensor devices and ease of preparation.
\end{abstract}

Lithographic outcomes on a surface are not only dependant on the instrumental parameters (e.g., loading force, orientation and scan speed), but they also depend on the different Young's modulus values of the polymer. It has been demonstrated that at high Young's modulus values ( $>5 \mathrm{GPa}$ ) no discernable lithographic outcomes have been achieved. At Young's modulus values in the low GPa range ( $<2 \mathrm{Gpa}$ ), wells, pits and orthogonal grids have be formed. On the other hand, softer polymer surfaces (Young's modulus in the $\mathrm{kPa}$ range) induced the stick-slip phenomena.

The stick-slip behaviour was observed in both the slow and fast direction of tip travel and was monitored during the manipulation process via friction loop analysis. After the manipulation process, the area was re-scanned using a soft lever revealing uniformly formed parallel channels. Friction loop analysis revealed a progression of the formation of the stick-slip mechanism in the fast scan direction with the lateral forces gradually increasing as the number of traverses increased. The stick-slip features then begin forming and progressing until the characteristic stick-slip features are observed. Just prior to the probe slipping in the slow scan direction, the stick-slip features in the fast scan direction break down completely. The tip then slips and assumes its next equilibrium position, repeating the cycle.
Lateral force data obtained on all three polymer surfaces and a calibration silicon surface showed the extent of in-plane deformation and frictional variation. The in-plane displacement (i.e., the vertical slope of the friction loop) was found to increase with a decrease in Young's modulus value which coincides with greater tip trapping, polymer deformation and relaxation.

By gradually increasing the loading force, the general trend of the lateral force was observed and plotted. The results are shown in figure 1 below. The lateral force increases linearly with an increase in loading force. As expected, the polyimide and silicon surfaces are comparable due to their Young's modulus values being > ca. 5 GPa. The P('BuMA) surface shows a greater increase with the PDMS sample showing the most dramatic increase. The three different linear responses for the PDMS surface are shown for the start, middle and end of a stick-slip cycle, with the final breakdown being omitted. A softer surface will lead to greater tip indentation, higher contact area, and therefore a higher lateral force. At higher loading forces, these effects are amplified.

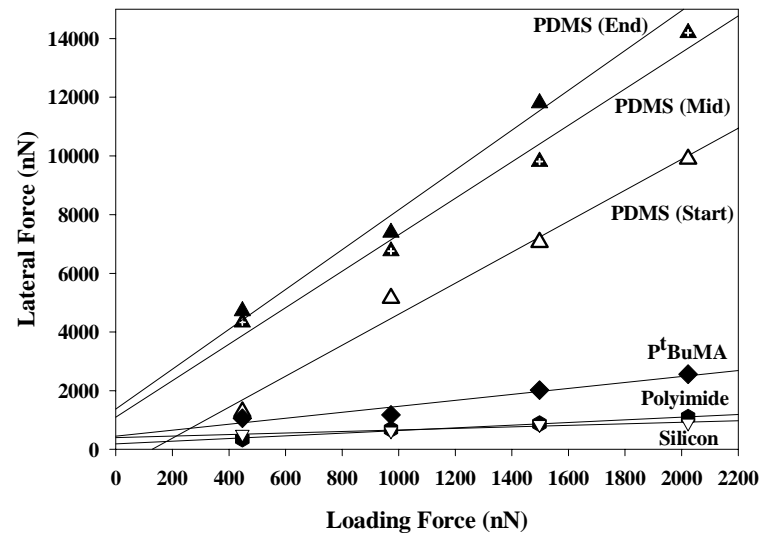

Figure 1 - Lateral force dependence on loading force for all four surfaces, with PDMS represented by the start, middle and end of the cycle. $R=0.691,0.929,0.857,0.948,0.994,0.961$ for silicon, polyimide, P('BuMA), PDMS (Start), PDMS (Mid) and PDMS (End), respectively. 
Respective coefficients of friction for the beginning, middle and end of the cycle on the PDMS surface were found to be ca. $5.3,6.2$ and 6.8, respectively. The coefficients of friction for the silicon, polyimide and $P\left({ }^{(} \mathrm{BuMA}\right)$ were ca. $0.26,0.46$ and 1 , respectively.

A direct comparison of the out-of-plane deformation of the PDMS and P('BuMA) surfaces was measured using the force-vsdistance mode. With an increasing loading force, the PDMS surface exhibited considerable indentation, e.g., load of $1500 \mathrm{nN}$, the surface indentation was ca. $2200 \mathrm{~nm}$. The $P\left({ }^{t} B u M A\right)$ reached an indentation limit of ca. $170 \mathrm{~nm}$ at a load of ca $320 \mathrm{nN}$.

Keywords - Lithography, Stick-Slip, Scanning Probe Microscopy, Manipulation, PDMS, P('BuMA), Polyimide.

\section{INTRODUCTION}

Polymers today are indispensable with their use exceeding that of steel, aluminium and copper combined. They offer many advantages in sensor technology over more traditional substrates such as gold. The ability to tailor surface chemistry, surface structure and other bulk and surface properties over a broad range accounts for their widespread use. Polymeric materials have also played important roles in the development of sensors due to their ability to increase analyte sensitivity and selectivity via phase-transfer chemistry. The ability for a polymer surface to promote or inhibit adhesion can be achieved by either coating, or chemically or physically altering the surface.

The (bio)chemical responses to biomaterials are associated with surface chemistry and structure rather than the bulk properties. The confinement of specific biospecies to particular regions on the surface is a major requirement for selectivity (e.g., array structures). The focus here is on the physical alteration/manipulation of three different polymer surfaces using the Scanning Probe Microscope (SPM) These polymers were chosen because of their availability, potential application as low cost biosensor devices and ease of preparation.

\section{EXPERIMENTAL DETAILS}

\section{A. Polymer Material Preparation}

Poly $\left({ }^{\text {tert }}\right.$ ButylMethacrylate) $\mathrm{P}\left({ }^{\mathrm{t}} \mathrm{BuMA}\right)$ (Polysciences, Inc.) was spin-coated (5 wt\% solution in cyclohexanone (Sigma Aldrich Co.)) at $3000 \mathrm{rpm}$, for $40 \mathrm{sec}$ on a Specialty Coating Systems spin coater (Model P6708) onto cover glass (grade \#1 thickness) pre-primed with hexamethyldisilazane (Sigma Aldrich Co.). The spin-coated substrates were then soft baked in a convection oven for 30 minutes at $90^{\circ} \mathrm{C}$ and stored in a desiccator prior to use.

The Polyimide (PI-2737) films were spin-coated onto atomically flat silicon wafer substrates, then allowed to cure at $350^{\circ} \mathrm{C}$ for 1 hour under an atmosphere of nitrogen.

Polydimethylsiloxane (PDMS) (Sylgard ${ }^{\circledR}$-184) was supplied by Dow Corning as a two part silicone elastomer. The base and curing agent were mixed at a 10:1 weight ratio, spin-coated onto atomically flat silicon wafers and cured in air $\left(25^{\circ} \mathrm{C}\right.$ and $55 \%$ relative humidity) for 48 hours prior to any analysis or manipulation.
A brief summary of some of the polymer specifications is shown in table 1 below.

TABLE $1 . \quad$ POLYMER SPECIFICATIONS

\begin{tabular}{|c|c|c|c|}
\hline $\begin{array}{c}\text { Properties }^{*} \\
\text { (As Cured) }\end{array}$ & P('BuMA) & $\begin{array}{c}\text { PI } 2730 \\
\text { Series }\end{array}$ & $\begin{array}{c}\text { Sylgard - } 184 \\
\text { PDMS }\end{array}$ \\
\hline Young's Modulus & $0.56 \mathrm{GPa}^{\mathrm{a}}$ & $5.4 \mathrm{GPa}^{\mathrm{b}}$ & $170-750 \mathrm{kPa}^{\mathrm{a}, \mathrm{c}}$ \\
\hline Tensile Strength & N/A & $178 \mathrm{MPa}$ & $7.10 \mathrm{MPa}$ \\
\hline Molecular Weight & $175 \mathrm{~K}$ & $\mathrm{~N} / \mathrm{A}$ & $95 \mathrm{~K}$ \\
\hline $\mathrm{Mw} / \mathrm{Mn}$ & 2.33 & N/A & 3.17 \\
\hline $\begin{array}{l}\text { Glass Transition } \\
\text { Temperature }\end{array}$ & $107^{\circ} \mathrm{C}$ & $>350^{\circ} \mathrm{C}$ & $<-120^{\circ} \mathrm{C}$ \\
\hline
\end{tabular}

\section{B. SPM Instrumentation and Probes}

The work was performed on two SPM instruments: a ThermoMicroscope TMX-2000 Explorer and a JEOL JSPM4200. Several scanners were used (JEOL JSPM-4200 - $25 \mu \mathrm{m}$ and $85 \mu \mathrm{m}$ tube scanners, with a z-range of ca $3 \mu \mathrm{m}$, and TermoMicroscope - $130 \times 130 \mu \mathrm{m}^{2}$ tripod scanner, with a zrange of $9.7 \mu \mathrm{m}$ ), in order to cover the scales of lateral, topographical and chemical differentiation. The lever-imposed loading force depended on the choice of lever stiffness. Forceversus-distance (F-d) curves were acquired at rates of translation in the $\mathrm{z}$-direction in the range $1-15 \mu \mathrm{ms}^{-1}$. Each $\mathrm{f}-\mathrm{d}$ curve consisted of 600 data points. F-d curves were obtained at randomly chosen locations within a typical field of view for averaging purposes.

The characteristics of the beam shaped levers (Ultrasharp NT-MDT) used are listed in table 2 below. Data for radius of curvature at the tip apex, $R_{\text {Tip }}$, $(<10 \mathrm{~nm})$ opening half-angle $\left(<10^{\circ}\right)$, and surface chemistry (native Si-oxide film) are summarized from the suppliers' specifications. The force constant for normal deflection, $k_{N}$, of individual probes were determined from the resonance method described by [4], and the torsional, $k_{T}$, and longitudinal, $k_{L}$, force constants were calculated from expressions described by [5].

TABLE $2 . \quad$ PROBE SPECIFICATIONS

\begin{tabular}{|l|l|l|l|}
\hline Probe & $\boldsymbol{k}_{\boldsymbol{N}} \mathbf{( \mathbf { N m } ^ { - 1 } )}$ & $\boldsymbol{k}_{\boldsymbol{T}} \mathbf{( \mathbf { N m } ^ { - 1 } )}$ & $\boldsymbol{k}_{\mathbf{L}} \mathbf{( \mathbf { N m } ^ { - 1 } )}$ \\
\hline A & 4.5 & 127 & 83 \\
\hline B & 9.2 & 200 & 103 \\
\hline C & 6.9 & 193 & 127 \\
\hline D & 0.1 & 15 & 10 \\
\hline E & 7.1 & 155 & 101 \\
\hline F & 10.3 & 222 & 145 \\
\hline G & 14 & 304 & 198 \\
\hline
\end{tabular}

\section{RESULTS AND DISCUSSION}

\section{A. Lithographic Outcomes}

Manipulation of the Polyimide surface is not possible as the surface is incompressible, having a Young's Modulus value ca. $5.4 \mathrm{GPa}$. Manipulation of the $\mathrm{P}\left({ }^{\mathrm{t}} \mathrm{BuMA}\right)$ surface resulted in the formation of various structures. Fig. 2(a) shows a topographical image of a rectangular pit formed as a result of a single raster scan at a normal force of $1 \mu \mathrm{N}$. The corresponding friction loop obtained when the tip traversed the region of the pit in (b) clearly shows a lower lateral force within the pit. Fig. 2(c) shows a grid pattern created as a platform for directional motion of molecular motors [6], with entry and exit points. The patterning can be applied to nanofluidic templates. 

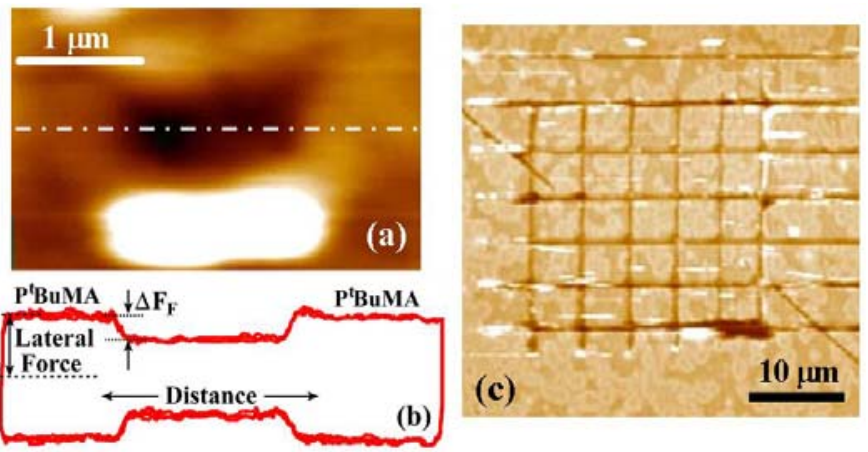

Figure 2 - A topographical image (a) showing a rectangular pit created using probe 'A'. (b) Friction loop corresponding to the line profile in (a) revealing a lower frictional force within the pit. (c) A grid pattern created with defined entry and exit points, using probe ' $\mathrm{B}$ ', produced as a platform for molecular motors [6].

Scanning at selected linear tip speeds and force loadings three-dimensional structures of arbitrary geometry can be constructed. Fig. 3(a) shows a 3-tiered well with terraces spaced at $\sim 15 \mathrm{~nm}$. The line profile in (b) shows relative heights of the 3 tiers.

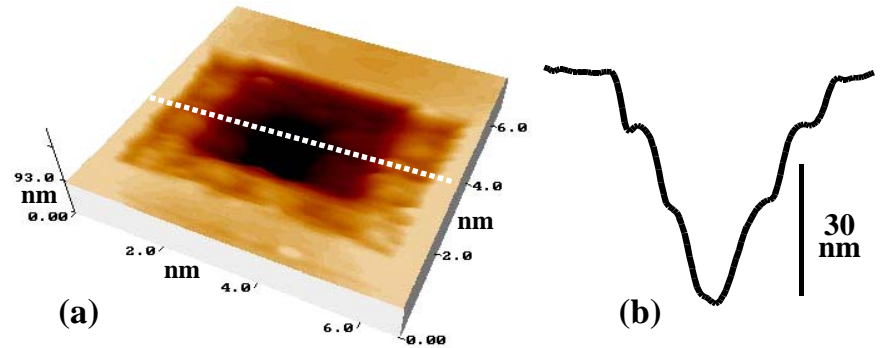

Figure 3 - (a) A three-dimensional terraced structure (probe 'C') formed by multiple scans at different force loadings and linear tip speeds. (b) A line profile of the structure.

\section{B. Stick-Slip Response}

Fig. 4(a) and (b) show a lateral force image and friction loop revealing the stick-slip responses in the slow and fast scan directions, respectively. The respective diagrammatic representations are shown in (c) and (d) [7]. The lateral force image in (a) was obtained using a soft lever (probe 'D') scanned after the manipulation of the PDMS surface using a stiff lever (probe ' $E$ '). The image clearly shows the series of channels and sloping lines connecting successive channels (arrows) indicating the stick and slip mechanism, respectively, in the slow scan direction. Fig. 4(c) is a diagrammatic representation of this event, where the tip begins its raster motion within a single stick point (solid horizontal grey band) at point A, scans across the surface in the fast scan direction, returns to its starting position (from point 1 to 2 in the inset), attempts to proceed down (see point 3 in the inset) and repeats this scanning procedure towards point $\mathrm{B}$. Once the buckling spring constant of the lever exceeds the constant of the restraining force, the tip slips into the next stick channel.

Fig. 4(b) shows a representative friction loop acquired during the scanning cycle, just prior to stick-slip breakdown, within a stick line in the fast scan direction (probe ' $F$ '). The beginning, middle and pre-breakdown loops have been discussed previously [7]. The vertical scale $\left(\Delta V_{x}\right)$ refers to the signal from the left-right (L-R) segments of the photo detector which monitors the torsional twisting of the lever. The $x$-stage displacement is monitored on the horizontal scale, with $\Delta x$ representing the in-plane deformation of the polymer surface, i.e., the extent to which the stick point is dragged along in response to the lever-imposed lateral force. The static friction shown in Fig. 4(b) is the point at which the motion of the tip and sample are coupled (for the polymer-tip contact). It is measured directly by monitoring the slope with respect to the vertical axis. The dynamic amplitude and spacing of the stickslip features are also defined. Fig. 4(d) shows a schematic representation of the stick-slip behaviour in the fast scan direction. As the tip moves across the surface ( $x$-direction) it becomes stuck at the first stick point (grey band) until the torsional force of the tip exceeds the stick force whereby the tip then slips to its next stick position, repeating the cycle in both the forward and reverse directions of travel.
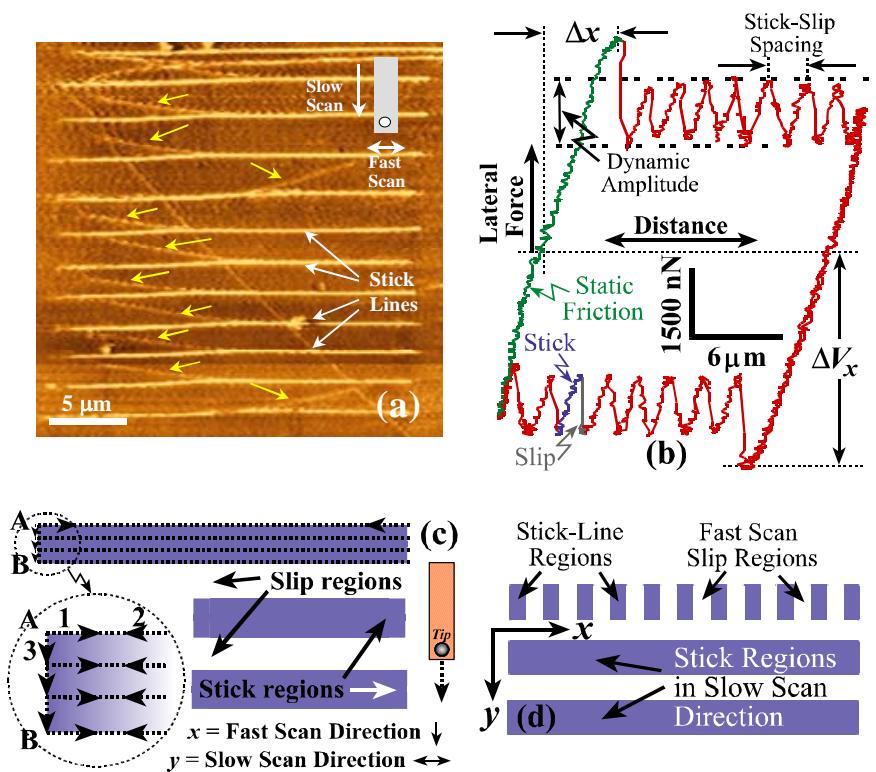

Figure 4 - (a) Lateral force image scanned over a manipulated region using a soft lever (probe ' $D$ ') showing the resulting stick lines and the path taken by the tip during the slip stage (arrows). (b) Friction loop obtained using probe ' $\mathrm{F}$ ' demonstrating stick-slip behaviour in the fast scan direction. $\Delta V_{x}$ represents the L-R detector signal with $\Delta x$ showing the lateral (in-plane) displacement of the stage. (c) A diagrammatic representation of the tip sticking, A to B (raster motion from 1 to 3 within a single gray band (channel) shown in the inset), then slipping through to the next region. (d) Schematic representation of the fast scan stick-slip motion of the lever.

\section{SPM-Based Polymer Analysis}

A comparison of the three polymer surfaces has been carried out. A loading force, $F_{\text {Load }}$, of $970 \mathrm{nN}$, applied by probe ' $\mathrm{G}$ ', at a scan speed of $125 \mu \mathrm{ms}^{-1}$ and at $300 \times 300$ lines of resolution was used. The three polymer surfaces, and a clean silicon surface were cut into small $0.5 \times 0.5 \mathrm{~cm}$ squares and placed on a stub. Fig. 5 shows a series of representative friction loops obtained during the scanning of the PDMS surface at the beginning, middle and end of the cycle, as well as data for the P( $\left.{ }^{\top} \mathrm{BuMA}\right)$, polyimide and silicon surfaces. The loops show an increase in lateral force, from 870 to $1400 \mathrm{nN}$ during the generation of a trough on the PDMS sample, while constant lateral forces of $320 \mathrm{nN}$ on the $\mathrm{P}\left({ }^{\mathrm{t}} \mathrm{BuMA}\right)$, and 160 
$\mathrm{nN}$ were measured for the polyimide and silicon surfaces. The friction/lateral data also show the varying degrees of in-plane deformation, with silicon and polyimide showing a vertical static friction slope indicative of an incompressible surface. The PDMS sample shows the characteristic stick-slip behaviour with the characteristic loss of stick-slip resolution towards the end of the cycle.

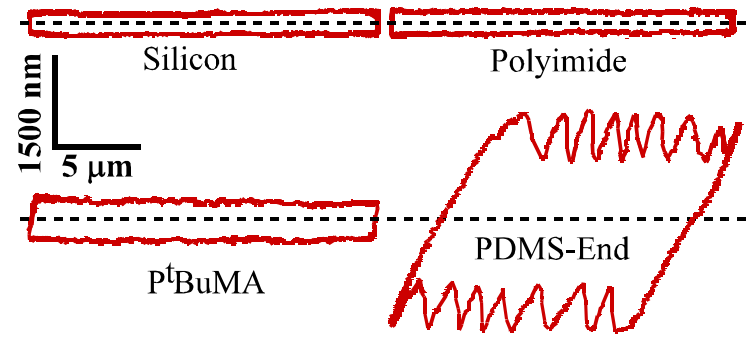

Figure 5 - Representative friction loops $\left(F_{\text {Load }}=970 \mathrm{nN}\right)$ using probe ' $\mathrm{G}$ ', taken on all four surfaces; PDMS, $\mathrm{P}\left({ }^{\mathrm{t}} \mathrm{BuMA}\right)$, polyimide and Silicon. The extent or absence of in-plane surface relaxation is also evident.

By gradually increasing the loading force, the general trend of the lateral force was observed and plotted. The results were obtained using porbe ' $G$ ' and are shown in Fig. 1 . The lateral force increases linearly with increasing loading force for all the surfaces, however, the absolute increase is quite different. Again, the polyimide and silicon surfaces are comparable, showing no resolvable differences. The P('BuMA) surface shows an increase, with the PDMS sample showing the most dramatic increase. In general a softer surface leads to greater tip indentation, higher contact area, and therefore a higher lateral force. These effects are amplified at higher loading forces.

The respective coefficients of friction for all of the polymer surfaces were also calculated. The PDMS has a range of coefficients of friction for the beginning, middle and end of the cycle of ca. 5.3, 6.2 and 6.8, respectively. The P( $\left.{ }^{\mathrm{t}} \mathrm{BuMA}\right)$, PI and silicon surfaces coefficients are ca. 1, 0.46 and 0.26 , respectively. The coefficient of friction for the silicon surface is slightly elevated when compared to the literature values of $0.09 \pm 0.03$ [8]. This is due to the tip being contaminated after the analysis on the PDMS and P( $\left.{ }^{\mathrm{t}} \mathrm{BuMA}\right)$ surfaces. The silicon surface was investigated with the tip after the other surfaces in order to reduce the risk of damaging/breaking the tip apex.

Fig. 6 shows a direct comparison of the out-of-plane deformation of the two 'soft' polymer surfaces under investigation, that is PDMS and $\mathrm{P}\left({ }^{\mathrm{t}} \mathrm{BuMA}\right)$, using the same tip and experimental conditions (probe ' $G$ '). Fig. 6 (a) shows a graphical relationship between the force $(\mathrm{nN})$ and indentation (nm), and (b) gives a direct f-d comparison between PDMS, $\mathrm{P}\left({ }^{\mathrm{t}} \mathrm{BuMA}\right)$ and the incompressible polyimide surface. The results clearly show the extent of compliance of the soft PDMS surface. As the force increases from $500 \mathrm{nN}$ to 1000 $\mathrm{nN}$ and $1500 \mathrm{nN}$, the PDMS surface indentation was ca. 1000 $\mathrm{nm}, 1850 \mathrm{~nm}$ and $2200 \mathrm{~nm}$, respectively. The P( $\left.{ }^{\mathrm{B}} \mathrm{BuMA}\right)$ indentation increased until a force ca. $320 \mathrm{nN}$, after which the indentation remained constant at ca. $170 \mathrm{~nm}$. The changes in contact areas for all the samples indicated by the indentation curves correlates well with the lateral responses.
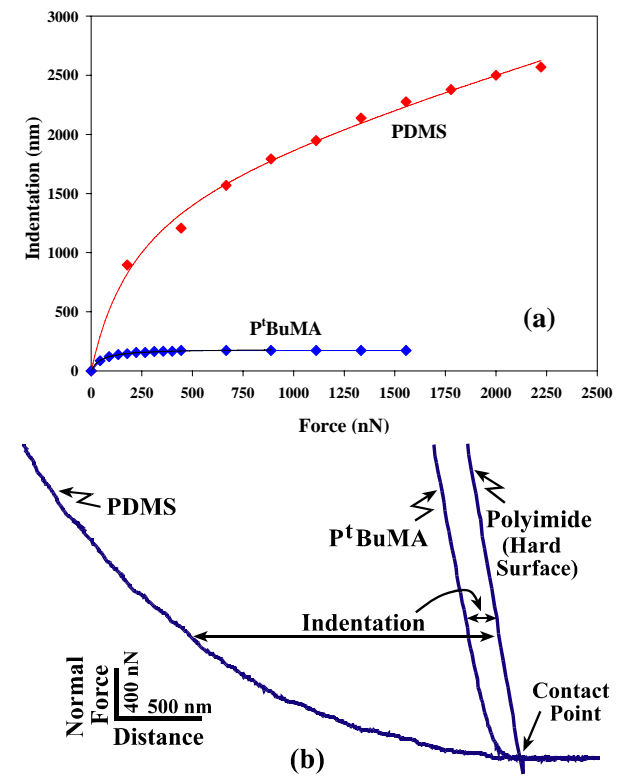

Figure 6 - (a) Force versus indentation curve comparing $\mathrm{P}\left({ }^{\mathrm{t}} \mathrm{BuMA}\right)$ and PDMS, and (b) representative f-d curves showing the extent of indentation using probe ' $\mathrm{G}$ '.

\section{CONCLUSION}

The manipulation of polymeric materials has been demonstrated to be dependant on the Young's Modulus values, scan speed and loading force. A polymer with a very low Young's modulus value will induce the stick-slip response, resulting in the formation of parallel channels. As well as a physical alteration to a polymer surface, there will also be possible chemical changes as seen from the friction loop data. Thus the effect of tip-induced surface manipulation is to alter the surface chemistry, presumably due to chain scission.

\section{REFERENCES}

[1] P. Roca-Cusachs, F. Rico, E. Martinez, J. Toset, R. Farre and D. Navajas, "Stability of microfabricated high aspect ration structures in poly(dimethylsiloxane),” Langmuir, vol. 21, pp. 5542-5548, 2005.

[2] D. Armani, C. Liu and N. Aluru, "Re-configurable fluid circuits by PDMS elastomer micromachining," $12^{\text {th }}$ Int. Conf. on MEMS (Orlando, FL) p. 222, 1998.

[3] M. A. Unger, J-P. Chou, T. Thorsen, A. Scherer and S. R. Quake, "Monolithic microfabricated valves and pumps by multilayer soft lithography," Science, vol. 288, pp. 113-116, 2000.

[4] J. P. Cleveland, S. Manne, D. Bocek and P. K. Hansma, “A nondestructive method for determining the spring constant of cantilevers for Scanning Force Microscopy,” Rev. Sci. Instr. Vol. 64, pp. 403-405, 1993.

[5] C. T. Gibson, G. S. Watson and S. Myhra, "Scanning Force Microscopy - Calibration procedures for 'best practice',” Scanning, vol. 19, pp. 564581, 1997.

[6] G. S. Watson, J. A. Blach, D. V. Nicolau, D. K. Pham, J. Wright and S. Myhra, "Surface topography and surface chemistry of radiationpatterned P(tBuMA) - Analysis by AFM, Polymer International, vol. 52, pp. 1408-1414, 2003.

[7] J. A. Watson, C. L. Brown, S. Myhra and G. S. Watson, "Twodimensional stick-slip on a soft elastic polymer: pattern generation using atomic force microscopy,” Nanotechnology, vol. 17, pp. 2581-2589, 2006.

[8] A. Crossley, C. Johnston, G. S. Watson and S. Myhra, "Tribology of Diamond-Like Carbon films from generic fabrication routes investigated by lateral force microscopy,” J. Phys. D, vol. 31, pp. 1955-1962, 1998. 